\title{
From stress to psychosis: whom, how, when and why?
}

\author{
V. Mondelli \\ Department of Psychological Medicine, King's College London, Institute of Psychiatry, London, UK
}

\begin{abstract}
The role of stress in precipitating the onset and relapse of psychosis has been now widely recognised. Over the past decade, the major challenge of research in this field has been to elucidate the biological mechanisms involved in the interaction between stress and psychosis onset. Obvious focus of this research has been the study of biological systems involved in the stress response. I will here briefly discuss the current evidence of abnormalities in stress biomarkers, such as cortisol and inflammatory markers, in patients with psychosis and their possible clinical implications. Stress biomarkers appear to hold strong potential as predictors of psychosis as well as of clinical outcome, and may represent optimal targets for the development of novel therapeutic agents for psychosis.
\end{abstract}

First published online 6 June 2014

Key words: Cortisol, inflammation, psychosis, stress.

The first clinical observations on the association between stress and mental disorders date back to the 18 th century, when the idea that social stress was one of the causes of mental illness was formulated in France by Philippe Pinel, and by his student Jean Esquirol. However, it was only later on that the first studies were conducted to specifically investigate the role of psychosocial stressful events in the onset of psychosis. The first major study investigating the potential relationship between the experience of stressful events and schizophrenia was conducted about 40 years ago (Brown \& Birley, 1968). This study reported that patients with schizophrenia experienced more stressful events in the 3 months preceding relapse of the illness than the control subjects. During the 3 weeks preceding the onset of the relapse, $46 \%$ of patients experienced at least one stressful event compared to $12 \%$ in an earlier 3 months period, suggesting a possible relationship between the life events and the onset of schizophrenia (Brown \& Birley, 1968). Some years later, Bebbington et al. (1993) replicated these findings, reporting an excess of stressful life events over the 6 months before the relapse of psychosis, both in patients with schizophrenia and in patients with affective psychoses when compared with a psychiatrically healthy sample from the local general population.

\footnotetext{
Address for correspondence: Dr V. Mondelli, Sections of Perinatal Psychiatry \& Stress, Psychiatry and Immunology (SPI-Lab), Centre for the Cellular Basis of Behaviour, The James Black Centre, Institute of Psychiatry, King's College London, 125 Coldharbour Lane, London SE5 9NU, UK.

(Email: valeria.mondelli@kcl.ac.uk)
}

These observations provided a basis for the diathesisstress model of schizophrenia, developed in the 1960-1970s. David Rosenthal played a central role in promulgating the view that the behavioural expression of the biological vulnerability for schizophrenia was influenced by exposure to stress. This view generated further research questions, mainly trying to clarify how exposure to stress could trigger the expression of constitutional vulnerability. Indeed, in the past decade, one of the major challenges in this research field has been to elucidate the biological mechanisms involved in the interaction between stress and onset of psychosis. Obvious focus of this research has been the study of biological systems involved in the stress response. I will discuss here some of the main findings on stress biomarkers in psychosis and their possible implications and translation in clinical settings.

\section{Hypothalamic-pituitary-adrenal (HPA) axis and psychosis}

The study of the HPA axis, the main biological system involved in the stress response, is central to reach a better understanding of the biological mechanisms behind the association between stress and psychosis and leading to the onset of psychosis. The HPA axis is activated by the release of corticotropin releasing hormone (CRH) and vasopressin (AVP), synthesised in the hypothalamus, which activate the secretion of adrenocorticotropic hormone (ACTH) from the pituitary, which finally stimulates the secretion of cortisol from the adrenal gland. Cortisol then interacts with its receptors in multiple target tissues also including the HPA axis, where it is responsible for the feedback 
inhibition of the secretion of ACTH from the pituitary and $\mathrm{CRH}$ from the hypothalamus (Pariante \& Lightman, 2008).

Studies conducted in the past decade have shown that patients with first-episode psychosis have increased activation in the HPA axis (elevated cortisol and ACTH levels, enlarged pituitary volume) (Walker et al. 2008; Mondelli et al. 2010a), together with a blunted cortisol awakening response (Mondelli et al. 2010a). The cortisol awakening response is a measure of acute reactivity of the HPA axis, so a blunted awakening response suggests reduced HPA axis reactivity to stress. This is consistent with the evidence that psychotic patients also have a blunted cortisol response to psychological and psychosocial stressors (Breier et al. 1988; Jansen et al. 1998). Within samples of patients with psychosis, reduced HPA axis reactivity to stress has been associated with more severe symptoms and worse cognitive function (Breier et al. 1988; Aas et al. 2011).

Interestingly, a hyperactivity of the HPA axis appears to be part of the biological vulnerability to psychosis, which is present prior to the onset of psychosis (Aiello et al. 2012). In particular, subjects who experience attenuated psychotic symptoms show increased cortisol levels as well as larger pituitary and smaller hippocampal volumes. Moreover, this HPA axis hyperactivity seems to be even greater among those individuals who subsequently develop frank psychosis. Similarly, relatives of patients with psychotic disorders present an increased sensitivity to stress, as shown by increased emotional reactivity to daily life stress, increased ACTH in response to stress, larger pituitary volume and smaller hippocampal volume (Aiello et al. 2012).

These abnormalities could play a relevant role in the development of psychosis through their effect on the neurotransmitter systems as well as on neurogenesis. In particular, administration of glucocorticoids has been shown to increase dopaminergic activity in the mesolimbic system in animals, suggesting a possible link between increased cortisol levels and the onset of psychotic symptoms (Schatzberg et al. 1985). Moreover, cortisol has been reported to blunt sympathetic nervous system responses activated by stress in humans (Raison \& Miller, 2003). Interestingly, although cortisol response to psychological stress is found to be blunted in first-episode psychosis, the sympathetic nervous system response to stress has been shown to be preserved in the same subjects (Van Venrooij et al. 2012). Therefore, it is possible to suggest that, in the presence of a stressful condition, the lack of cortisol response cannot restrain the activation of the sympathetic nervous system, resulting in a persistent increased arousal and a consequent exacerbation of psychotic symptoms (Borges et al. 2013).
The effect of HPA axis activation on neurogenesis and neuroplasticity is one of the other mechanisms suggested explaining the association between abnormalities in HPA axis and onset of psychosis. Indeed, glucocorticoids can also influence neuroplasticity (decreasing neurogenesis and remodelling neuronal dendrites), affecting neurotrophins levels, such as brain-derived neurotrophic factor, and through their interaction with pro-inflammatory cytokines, excitatory amino acid neurotransmitters and N-methyl-Daspartate (NMDA) receptors (McEwen, 2000). This is particularly important since a number of studies have shown brain volume changes at the onset of psychosis, or during the transition to psychosis, suggesting a critical role for neuroplasticity, especially in specific brain areas, in the development of psychosis (Cahn et al. 2009; Takahashi et al. 2009). Indeed, we have shown that high cortisol levels are associated with smaller hippocampal volume in first-episode psychosis, further supporting this biological pathway to explain the link between HPA axis hyperactivity and the onset of psychosis (Mondelli et al. 2010b, 2011).

Interestingly, antipsychotic medications can affect cortisol levels, but have been reported to be able to only partly re-establish a 'normal' HPA axis activity; in particular antipsychotic treatment seem to reduce/ normalise the high levels of cortisol during the day but does not seem to affect the abnormalities in the cortisol awakening response (Mondelli et al. 2010a). Furthermore, persistent non-suppression of cortisol levels following the dexamethasone test after 4 weeks of antipsychotic treatment is associated with a poor clinical outcome (Tandon et al. 1991). Conversely, a reduction in cortisol levels after 12 weeks of antipsychotic treatment is associated with an improvement in psychotic symptoms (Zhang et al. 2005; Garner et al. 2011). Further studies would need to investigate if the regulation of the HPA axis is important for response to treatment, and if we can use the HPA axis as a possible biological target system for the development of novel drugs for treatment-resistant patients.

\section{Inflammation and psychosis}

The immune system has also been demonstrated to be one of the biological systems activated by psychosocial stress; and indeed recent studies suggest inflammation as one of the possible mediators between stress and the onset of psychiatric disorders. More specifically, when we mention inflammation in this context we refer to a phenomenon called systemic or chronic, low-grade inflammation which is different from responses to acute infectious stimuli because (1) it is systemic and not limited to a local site of injury or infection; (2) it 
is of lower magnitude than other types of inflammation; (3) it is typically a longer term phenomenon, as opposed to the transient nature of inflammatory responses to infection; and (4) does not have a clear origin or does not follow an apparent stimulus, such as infection or injury (Rohleder, 2014).

Over the past few decades of research, there has been an incredibly increasing body of enquiry supporting the role of chronic, low-grade inflammation in the pathogenesis of different psychiatric disorders from both preclinical and clinical studies (Dantzer, 2001; Dantzer et al. 2008). The most consistent and established findings come from studies in depression. However, over the past few years, it has become more and more evident that an increased inflammatory status is present both at the onset of psychosis and in the later stages of the disorder. This has been suggested by findings of increased cytokine levels in peripheral blood and cerebrospinal fluid in patients with psychosis (Miller et al. 2011; Zajkowska \& Mondelli, 2014). In particular, increased levels of the pro-inflammatory cytokines interleukin (IL)-6, tumour necrosis factor- $\alpha$ and IL- $1 \beta$ are the most consistent findings from the studies conducted in firstepisode psychosis patients (Zajkowska \& Mondelli, 2014). The mechanisms through which inflammatory cytokines have been suggested to mediate the onset of psychosis include interaction with multiple pathways such as monoamine metabolism, neuroendocrine function and synaptic plasticity. Interestingly, increased inflammation appears to contribute to the brain abnormalities evident at the onset of psychosis, as shown by our previous findings of an association between increased levels IL-6 and reduced hippocampal volume in first-episode psychosis patients (Mondelli et al. 2011).

Studies on the effect of antipsychotic medication on inflammatory markers have given so far mixed findings, showing both anti-inflammatory and pro-inflammatory effects. Indeed, some of these discrepancies could be partly explained by what reported by a recent longitudinal study showing an initial anti-inflammatory effect of antipsychotic medication followed by a gradual rise again of cytokines levels along an increase in body weight (Song et al. 2014). These data suggest that the presence of both a direct anti-inflammatory activity of the antipsychotic treatment and an indirect pro-inflammatory activity, possibly mediated by their effect on weight-gain and increased adiposity. It remains, however, unclear whether the possible anti-inflammatory effect of these medications contributes to improve the clinical outcome of these patients. Interestingly, only three studies have investigated so far whether the increased inflammation is linked to clinical outcomes in psychosis, and all found that higher inflammation was associated with a poorer clinical outcome (McAllister et al. 1995; Lin et al. 1998; Zhang et al. 2005).

\section{Clinical implications and conclusions}

Our understanding of the biological mechanisms linking stress to psychosis onset has greatly increased over the past few years. Indeed, we have now a clearer picture of what are the biological abnormalities in the stress response present in patients with psychosis. But how can we use this new knowledge about stress biomarkers to improve clinical care of patients with psychosis? Can we use stress biomarkers to predict who is going to develop psychosis following exposure to high levels of stress? Can we use stress biomarkers to predict who is going to be resistant to antipsychotic treatment? Are biological systems involved in the stress response a new therapeutic target for patients with psychosis? The findings so far suggest that stress biomarkers hold strong potential as predictors of psychosis as well as of clinical outcome, and may represent optimal targets for the development of novel therapeutic agents. Future studies will be fundamental to reveal the clinical benefit of all the research work conducted in the last 40 years on the link between stress and psychosis.

\section{Acknowledgements}

This work has been supported by the South London and Maudsley NHS Foundation Trust \& Institute of Psychiatry NIHR Biomedical Research Centre for Mental Health.

\section{Financial Support}

This work has been supported by the South London and Maudsley NHS Foundation Trust \& Institute of Psychiatry NIHR Biomedical Research Centre for Mental Health.

\section{Conflict of Interest}

None.

\section{Ethical Standard}

Not applicable.

\section{References}

Aas M, Dazzan P, Mondelli V, Toulopoulou T, Reichenberg A, Di Forti M, Fisher HL, Handley R, Hepgul N, Marques T, Miorelli A, Taylor H, Russo M, Wiffen B, Papadopoulos A, Aitchison KJ, Morgan C, Murray RM, Pariante CM (2011). Abnormal cortisol awakening response predicts worse cognitive function in patients with first-episode psychosis. Psychological Medicine 41, 463-476.

Aiello G, Horowitz M, Hepgul N, Pariante CM, Mondelli V (2012). Stress abnormalities in individuals at risk for psychosis: a review of studies in subjects with familial risk or with "at risk" mental state. Psychoneuroendocrinology 37, 1600-1613. 
Bebbington P, Wilkins S, Jones P, Foerster A, Murray R, Toone B, Lewis S (1993). Life events and psychosis: initial results from the Camberwell Collaborative Psychosis Study. British Journal of Psychiatry 162, 72-79.

Borges S, Gayer-Anderson C, Mondelli V (2013). A systematic review of the activity of the hypothalamicpituitary-adrenal axis in first episode psychosis.

Psychoneuroendocrinology 38, 603-611.

Breier A, Wolkowitz OM, Doran AR, Bellar S, Pickar D (1988). Neurobiological effects of lumbar puncture stress in psychiatric patients and healthy volunteers. Psychiatry Research 25, 187-194.

Brown GW, Birley JL (1968). Crises and life changes and the onset of schizophrenia. Journal of Health and Social Behavior 9, 203-214.

Cahn W, Rais M, Stigter FP, Van Haren NE, Caspers E, Hulshoff Pol HE, Xu Z, Schnack HG, Kahn RS (2009). Psychosis and brain volume changes during the first five years of schizophrenia. European Neuropsychopharmacology 19, 147-151.

Dantzer R (2001). Cytokine-induced sickness behavior: mechanisms and implications. Annals of the New York Academy of Sciences 933, 222-234.

Dantzer R, O'Connor JC, Freund GG, Johnson RW, Kelley KW (2008). From inflammation to sickness and depression: when the immune system subjugates the brain. Nature Reviews Neuroscience 9, 46-56.

Garner B, Phassouliotis C, Phillips LJ, Markulev C, Butselaar F, Bendall S, Yun Y, Mcgorry PD (2011). Cortisol and dehydroepiandrosterone-sulphate levels correlate with symptom severity in first-episode psychosis. Journal of Psychiatric Research 45, 249-255.

Jansen LM, Gispen-de Wied CC, Gademan PJ, De Jonge RC, Van Der Linden JA, Kahn RS (1998). Blunted cortisol response to a psychosocial stressor in schizophrenia. Schizophrenia Research 33, 87-94.

Lin A, Kenis G, Bignotti S, Tura GJ, De Jong R, Bosmans E, Pioli R, Altamura C, Scharpe S, Maes M (1998). The inflammatory response system in treatment-resistant schizophrenia: increased serum interleukin-6. Schizophrenia Research 32, 9-15.

Mcallister CG, Van Kammen DP, Rehn TJ, Miller AL, Gurklis J, Kelley ME, Yao J, Peters JL (1995). Increases in CSF levels of interleukin-2 in schizophrenia: effects of recurrence of psychosis and medication status. American Journal of Psychiatry 152, 1291-1297.

Mcewen BS (2000). The neurobiology of stress: from serendipity to clinical relevance. Brain Research 886, 172189.

Miller BJ, Buckley P, Seabolt W, Mellor A, Kirkpatrick B (2011). Meta-analysis of cytokine alterations in schizophrenia: clinical status and antipsychotic effects. Biological Psychiatry 70, 663-671.

Mondelli V, Dazzan P, Hepgul N, Di Forti M, Aas M, D'Albenzio A, Di Nicola M, Fisher H, Handley R, Marques TR, Morgan C, Navari S, Taylor H, Papadopoulos A, Aitchison KJ, Murray RM, Pariante CM (2010a). Abnormal cortisol levels during the day and cortisol awakening response in first-episode psychosis: the role of stress and of antipsychotic treatment. Schizophrenia Research 116, 234-242.

Mondelli V, Pariante CM, Navari S, Aas M, D'Albenzio A, Di Forti M, Handley R, Hepgul N, Marques TR, Taylor H, Papadopoulos AS, Aitchison KJ, Murray RM, Dazzan P (2010b). Higher cortisol levels are associated with smaller left hippocampal volume in first-episode psychosis.

Schizophrenia Research 119, 75-78.

Mondelli V, Cattaneo A, Belvederi Murri M, Di Forti M, Handley R, Hepgul N, Miorelli A, Navari S, Papadopoulos AS, Aitchison KJ, Morgan C, Murray RM, Dazzan P, Pariante CM (2011). Stress and inflammation reduce brain-derived neurotrophic factor expression in first-episode psychosis: a pathway to smaller hippocampal volume. Journal of Clinical Psychiatry 72, 1677-1684.

Pariante CM, Lightman SL (2008). The HPA axis in major depression: classical theories and new developments. Trends in Neurosciences 31, 464-468.

Raison CL, Miller AH (2003). When not enough is too much: the role of insufficient glucocorticoid signaling in the pathophysiology of stress-related disorders. American Journal of Psychiatry 160, 1554-1565.

Rohleder N (2014). Stimulation of systemic low-grade inflammation by psychosocial stress. Psychosomatic Medicine 76, 181-9.

Schatzberg AF, Rothschild AJ, Langlais PJ, Bird ED, Cole JO (1985). A corticosteroid/dopamine hypothesis for psychotic depression and related states. Journal of Psychiatric Research 19, 57-64.

Song X, Fan X, Li X, Zhang W, Gao J, Zhao J, Harrington A, Ziedonis D, Luxian Lv (2014). Changes in pro-inflammatory cytokines and body weight during 6-month risperidone treatment in drug naive, first-episode schizophrenia. Psychopharmacology 231, 319-325.

Takahashi T, Wood SJ, Yung AR, Soulsby B, Mcgorry PD, Suzuki M, Kawasaki Y, Phillips LJ, Velakoulis D, Pantelis C (2009). Progressive gray matter reduction of the superior temporal gyrus during transition to psychosis. Archives of General Psychiatry 66, 366-376.

Tandon R, Mazzara C, Dequardo J, Craig KA, Meador-Woodruff JH, Goldman R, Greden JF (1991). Dexamethasone suppression test in schizophrenia: relationship to symptomatology, ventricular enlargement, and outcome. Biological Psychiatry 29, 953-964.

Van Venrooij JA, Fluitman SB, Lijmer JG, Kavelaars A, Heijnen CJ, Westenberg HG, Kahn RS, Gispen-De Wied CC (2012). Impaired neuroendocrine and immune response to acute stress in medication-naive patients with a first episode of psychosis. Schizophrenia Bulletin 38, 272-279.

Walker E, Mittal V, Tessner K (2008). Stress and the hypothalamic pituitary adrenal axis in the developmental course of schizophrenia. Annual Review of Clinical Psychology 4, 189-216.

Zajkowska Z, Mondelli V (2014). First-episode psychosis: an inflammatory state? Neuroimmunomodulation 21, 102-108.

Zhang XY, Zhou DF, Cao LY, Wu GY, Shen YC (2005). Cortisol and cytokines in chronic and treatment-resistant patients with schizophrenia: association with psychopathology and response to antipsychotics. Neuropsychopharmacology 30, 1532-1538. 